\section{Prevalência de distúrbios \\ osteomusculares relacionados ao trabalho em profissionais de enfermagem}

\author{
Prevalence of musculoskeletal \\ disorders in nursing professionals
}

Natália Fonseca Ribeiro'

Rita de Cássia Pereira Fernandes'

Davi Jorge Fontoura Solla"

Anivaldo Costa Santos Junior"

Antonio Santos de Sena Junior"

' Programa De Pós-graduação em Saúde, Ambiente e Trabalho da Faculdade de Medicina da Bahia - Universidade Federal da Bahia.

" Departamento de Medicina Preventiva e Social - Faculdade de Medicina da Bahia - Universidade Federal da Bahia.

Estudo financiado pelo Conselho Nacional de Desenvolvimento Científico e Tecnológico - CNPQ. Processo № 42176/2008-0.

Correspondência: Natália da Rosa Fonseca. Largo do Terreiro de Jesus, s/n, Centro Histórico, Salvador, Bahia, CEP 40.026-010. E-mail: fonseca.natalia@bol.com.br

\section{Resumo}

Estudo de corte transversal estimou a prevalência de distúrbios osteomusculares relacionados ao trabalho em auxiliares e técnicas de enfermagem em Salvador, Bahia. Trezentas e oito trabalhadoras, selecionadas aleatoriamente, responderam um questionário aplicado por entrevistadores treinados durante o expediente de trabalho. A maioria das entrevistadas trabalhava no turno diurno e não costumava fazer hora extra. Cerca de $34 \%$ relataram ter outra atividade regular remunerada e o tempo médio no mercado de trabalho formal ou informal foi de 19 anos. Verificou-se alta exposição ocupacional a movimentos repetitivos com as mãos, posturas de trabalho em pé e andando, posturas inadequadas do tronco e levantamento de carga. A prevalência de DORT em pelo menos um segmento corporal foi de $83,4 \%$. As principais regiões corporais acometidas foram: lombar $(53,9 \%)$, pernas $(51,9 \%)$, pescoço $(36,4 \%)$, parte alta do dorso $(35,7 \%)$ e ombros $(33,8 \%)$. Verificou-se elevada prevalência de DORT na população estudada, o que aponta para necessidade de melhoria nas condições de trabalho dessas profissionais.

Palavras-chave: Distúrbios osteomusculares relacionados ao trabalho. Lesões por esforços repetitivos. DORT. Enfermagem. Auxiliares de enfermagem. 


\section{Abstract}

A cross-sectional study estimated the prevalence of work-related musculoskeletal disorders among nursing assistants in Salvador, Bahia. Three hundred and eight workers, randomly selected, answered a questionnaire applied by trained interviewers during working hours. The majority of respondents worked the day shift and did not usually work overtime. About $34 \%$ reported having another regular job. Average time in the formal or informal labor market was 19 years. There was high occupational exposure to repetitive hand movements, standing posture, walking, inadequate postures of the trunk and manual handling of loads. The prevalence of work-related musculoskeletal disorders in at least one body segment was 83.4\%.The most affected body segments were: low back (53.9\%), legs (51.9\%), neck (36.4\%), upper back (35.7\%) and shoulders $(33.8 \%)$. There was high prevalence of work-related musculoskeletal disorders in the studied population, which points out to the need for improvements in the working conditions of those professionals.

Keywords: Cumulative trauma disorders. Repetition strain injury. Musculoskeletal disorders. Nursing. Nursing assistants.

\section{Introdução}

Grande parte dos profissionais de saúde atua em unidades hospitalares, sendo que a maioria desses trabalhadores corresponde a profissionais de enfermagem. No ambiente hospitalar, a prioridade é dada ao conforto e à segurança do usuário; no entanto, os trabalhadores que compartilham desse ambiente podem sofrer alterações de saúde, entre as quais se destacam os distúrbios osteomusculares relacionados ao trabalho (DORT) ${ }^{1}$.

Os DORT, termo que abrange um grupo heterogêneo de distúrbios do sistema musculoesquelético com variado quadro clínico, incluindo queixas de dor, formigamento, dormência, peso e fadiga precoce ${ }^{2}$, são um dos problemas mais frequentes $\mathrm{e}$ mais dispendiosos entre profissionais de enfermagem, contribuindo significativamente para a incapacidade e o afastamento do trabalho ${ }^{3}$.

As posturas inadequadas exigidas durante a prestação de cuidados, alguns aspectos da organização do trabalho (como, por exemplo, o trabalho por turnos e o elevado número de doentes a ser atendido), bem como as características morfológicas dos pacientes e a inadequada configuração arquitetônica dos locais de trabalho, entre outros, são elementos que contribuem para explicar o desenvolvimento dessas lesões ${ }^{4}$.

Diversos estudos em todo o mundo têm destacado a elevada morbidade por DORT entre profissionais de enfermagem ${ }^{1,4-7}$. Entretanto, não foi encontrado estudo epidemiológico sobre os DORT entre profissionais de enfermagem no estado da Bahia. Assim, planejou-se este estudo com o objetivo de conhecer as demandas físicas e psicossociais às quais estão expostas técnicas e auxiliares de enfermagem de um hospital público de Salvador-BA, como também estimar a prevalência de DORT entre essas profissionais.

\section{Métodos}

Foi realizado um estudo de corte transversal com técnicas e auxiliares de 
enfermagem (TAE) de um hospital público, referência para atendimento de emergência/urgência, na cidade de Salvador, BA. As TAE compõem um grupo numeroso $\mathrm{e}$ estão expostas a uma maior demanda física no trabalho do que outros profissionais de saúde.

Esse hospital possuía, na época da coleta de dados (primeiro trimestre de 2008), 666 TAE em seu quadro de servidores. $\mathrm{O}$ tamanho mínimo da amostra, 293 indivíduos, foi calculado considerando-se um grau de precisão absoluta de 5,0\%, nível de confiança de $95,0 \%$, prevalência esperada de $50,0 \%$ e efeito de desenho de 1,2. Prevendo as perdas que poderiam ocorrer, o tamanho da amostra foi ampliado para 320 indivíduos.

Foram elegíveis para o estudo todas as TAE em efetivo exercício profissional no momento da coleta dos dados na unidade hospitalar selecionada, de acordo com listagem fornecida pela instituição. As servidoras foram selecionadas aleatoriamente para compor a amostra. Aquelas selecionadas que se encontravam afastadas do trabalho foram substituídas pelo próximo nome da lista.

Para a coleta de dados foi aplicado um questionário às trabalhadoras, durante o expediente de trabalho, em local reservado. Esse instrumento é uma adaptação do questionário elaborado e utilizado por Fernandes ${ }^{8}$ para o estudo de DORT em trabalhadores da indústria de plástico, em que se utilizaram questões elaboradas pela autora e questões retiradas de outros instrumentos, modificadas ou não.

O questionário contém questões sobre aspectos sociodemográficos, história ocupacional no emprego atual e vida laboral pregressa, demandas físicas e psicossociais no trabalho, uso de fumo, bebidas alcoólicas e contraceptivo hormonal, atividades domésticas, e questões sobre DORT. Foram ainda coletados o peso e altura referidos pelas TAE para o cálculo do Índice de Massa Corporal (IMC). Obesidade foi considerada como IMC maior ou igual $30 \mathrm{~kg} / \mathrm{m}^{2}$ e sobrepeso como IMC entre 25 e $29,9 \mathrm{~kg} / \mathrm{m}^{2}$.

Os dados sobre a demanda física no trabalho foram obtidos por meio de questões respondidas pelas trabalhadoras em escala de frequência, intensidade ou duração de 0 a 5, com âncoras nas extremidades, sobre posturas de trabalho, movimentos repetitivos com as mãos, força muscular exercida, levantamento de cargas e manuseio de pacientes.

Os aspectos psicossociais do trabalho foram medidos pelo Job Content Questionnaire (JCQ $)^{9}$, por meio dos escores obtidos para demanda psicológica, controle, suporte social e insatisfação no trabalho. Estudos nacionais sobre o desempenho do JCQ relataram bons indicadores de validade e de confiabilidade ${ }^{10,11}$.

A demanda psicológica caracteriza-se pelas exigências que o trabalhador enfrenta no seu trabalho, seja quanto à concentração, ritmo ou tempo para realização das tarefas. O controle considera a possibilidade de o trabalhador tomar decisões acerca do seu trabalho, como também o nível de habilidade ou criatividade requerido para a tarefa e a flexibilidade que permite ao trabalhador decidir quais habilidades empregar. Já o suporte social abarca a colaboração e o apoio proveniente da chefia e dos colegas de trabalho ${ }^{12}$. Essas variáveis foram dicotomizadas utilizando-se a mediana como ponto de corte.

Os dados sobre sintomas de DORT foram coletados por meio da versão ampliada do Nordic Musculoskeletal Questionnaire (NMQ), instrumento amplamente utilizado em investigações epidemiológicas sobre DORT em todo o mundo. Avalia-se a presença de dor ou desconforto nos últimos 12 meses nas áreas anatômicas estudadas, e sua severidade, duração e frequência. Estudos demonstram boa validade ${ }^{13} \mathrm{e}$ confiabilidade ${ }^{14}$ da versão brasileira do NMQ.

"Caso de DORT" foi definido por: dor ou desconforto em uma ou mais das seguintes áreas corporais: dedos, punhos, mãos, antebraços, cotovelos, pescoço, ombro, região alta do dorso, região lombar, coxas, joelhos, pernas, tornozelos ou pés, nos últimos doze meses, com duração mínima de uma semana ou frequência mínima mensal, não 
decorrente de trauma agudo, acompanhados de pelo menos um dos seguintes sinais de gravidade:

- grau de severidade maior ou igual a três, em uma escala numérica de zero a cinco com âncoras nas extremidades (nenhum desconforto a desconforto insuportável);

- busca de atenção médica pelo problema;

- ausência ao trabalho (oficial ou não);

- mudança de trabalho por restrição de saúde ${ }^{15}$.

A equipe de entrevistadores, formada pela autora principal e estudantes do curso de graduação em Medicina da Universidade Federal da Bahia, foi treinada previamente para o uso do instrumento, e esclarecida acerca de cada item do questionário e das alternativas de resposta.

Este projeto de pesquisa foi aprovado por um Comitê de Ética em Pesquisa pelo parecer no 84/2007 de 23 de maio de 2007. Antes da aplicação dos questionários, as trabalhadoras foram informadas dos objetivos da pesquisa, da instituição responsável e de que o hospital apenas liberou o acesso dos pesquisadores à instituição, não tendo qualquer participação na realização da pesquisa. As trabalhadoras selecionadas que aceitaram participar do estudo assinaram um Termo de Consentimento Livre e Esclarecido, onde foram assegurados o sigilo das informações, a participação voluntária e o anonimato.

\section{Resultados}

Das 320 TAE selecionadas, 308 (96,2\%) participaram do estudo. A média de idade das entrevistadas foi de 41 anos, com desvio padrão de 11 anos. A maioria das entrevistadas $(97,4 \%)$ possuía ao menos o ensino médio completo, sendo que, destas, $8,1 \%$ possuíam ainda o ensino superior completo. Cerca de $50 \%$ das trabalhadoras eram casadas, oficialmente ou não, e apenas $5,5 \%$ possuíam filhos menores de dois anos. Cerca de $61,7 \%$ das entrevistadas eram naturais de Salvador (Tabela 1).

\section{[INSERIR A TABELA 1]}

O hábito de fumar estava presente em apenas $8,8 \%$ das trabalhadoras e o consumo de bebidas alcoólicas, com frequência de uma vez por semana ou mais, foi relatado por $19,5 \%$ da população estudada. De acordo com o IMC, $49 \%$ das trabalhadoras apresentavam sobrepeso ou obesidade (Tabela 1).

Em relação à unidade de trabalho, 22,1\% das entrevistadas estavam inseridas na emergência, $14,3 \%$ na unidade de terapia intensiva (UTI), 11,4\% no centro cirúrgico e as demais se distribuíam entre os outros setores do hospital. Aproximadamente $53 \%$ das TAE já mudaram de setor dentro do hospital e, destas, $23 \%$ o fizeram por motivo de saúde. Entre os problemas que motivaram as mudanças de setor, os mais frequentes foram os relacionados à coluna. A UTI e a emergência foram os setores onde se constatou o maior número de mudanças por motivo de saúde.

A maioria das entrevistadas trabalhava no turno diurno e não costumava fazer hora extra $(56,5 \%$ e $74,4 \%$, respectivamente). Cerca de $34,1 \%$ relataram ter outra atividade regular remunerada, dentre as quais, $82,9 \%$ desempenhavam também a função de TAE nesse outro trabalho (Tabela 2).

O tempo médio no mercado de trabalho formal ou informal foi de 19 anos $( \pm 10,7)$. A maioria das entrevistadas (63\%) sempre trabalhou como TAE, enquanto $37 \%$ já haviam trabalhado em outras funções. Destas últimas, $22,8 \%$ já tiveram outras ocupações no setor saúde e $27,2 \%$ já desempenharam atividades no comércio. A média de horas de trabalho semanais no hospital foi de 32 horas e de trabalho doméstico de $18 \mathrm{~h}$ (Tabela 2).

O auto-relato das TAE sobre exposição às demandas físicas no trabalho revela alta exposição a movimentos repetitivos com as mãos. Além disso, constatou-se que a maioria das trabalhadoras adotava as posturas em pé e andando durante a maior parte do tempo, enquanto a postura sentada mostrou-se pouco comum entre 
Tabela 1 - Características sociodemográficas e de hábitos de vida de técnicas e auxiliares de enfermagem. Salvador, 2008.

Table 1 - Socio-demographic and life habit characteristics of nursing assistants. Salvador, 2008.

\begin{tabular}{|c|c|}
\hline Variáveis & Frequência \\
\hline Idade (anos, média $\pm \mathrm{dp}$ ) & $41 \pm 11,1$ \\
\hline \multicolumn{2}{|l|}{ Estado civil n (\%) } \\
\hline Casado ou vive junto & $155(50,3)$ \\
\hline Solteiro & $109(35,4)$ \\
\hline \multicolumn{2}{|l|}{ Tem filho(s) n (\%) } \\
\hline$<2$ anos & $17(5,5)$ \\
\hline$\geq 2$ anos & $191(62,0)$ \\
\hline Não tem filhos & $100(32,5)$ \\
\hline \multicolumn{2}{|l|}{ Escolaridade n (\%) } \\
\hline < Ensino Médio & $8(2,6)$ \\
\hline Ensino Médio & $239(77,6)$ \\
\hline Superior incompleto & $36(11,7)$ \\
\hline Superior Completo & $25(8,1)$ \\
\hline \multicolumn{2}{|l|}{ Naturalidade n (\%) } \\
\hline Salvador & $190(61,7)$ \\
\hline Interior da Bahia & $100(32,5)$ \\
\hline Outros estados & $18(5,8)$ \\
\hline \multicolumn{2}{|c|}{ Uso de bebida alcoólica n (\%) } \\
\hline$\geq 1 \mathrm{vez} /$ semana & $60(19,5)$ \\
\hline$<1 \mathrm{vez} /$ semana & $248(80,5)$ \\
\hline \multicolumn{2}{|l|}{ Hábito de fumar n(\%) } \\
\hline Sim & $27(8,8)$ \\
\hline Não & $281(91,2)$ \\
\hline \multicolumn{2}{|c|}{ Índice de Massa Corporal n (\%) } \\
\hline Baixo peso & $23(7,6)$ \\
\hline Normal & $132(43,4)$ \\
\hline Sobrepeso/obesidade & $174(49,0)$ \\
\hline
\end{tabular}

as trabalhadoras. O levantamento de carga e a força muscular desenvolvida com os braços e com as mãos também ocupavam a maior parte da jornada de trabalho das TAE estudadas. As posturas inadequadas, como o tronco inclinado para frente ou rodado, são comuns no cotidiano das TAE.

Na Tabela 3 estão mostrados os escores obtidos para demanda psicológica, controle, suporte social e insatisfação no trabalho. Constatou-se que as TAE da emergência foram as que obtiveram escores mais altos para controle sobre o trabalho, seguidas pelas TAE das enfermarias (ortopedia, clinica cirúrgica alas A e B, e enfermaria de traumatismo raquimedular) e o mais baixo controle foi encontrado no Centro de Material Esterilizado (CME). O mais alto escore para demanda psicológica foi encontrado no Centro Cirúrgico, e o maior escore para suporte social no Centro de Tratamento de Queimados (CTQ) e a maior insatisfação no trabalho nas enfermarias.

A prevalência dos DORT em pelo menos um segmento corporal foi de $83,4 \%$. Considerando-se as regiões corporais de forma isolada, constatam-se as mais elevadas prevalências nas seguintes regiões: lombar $(53,9 \%)$, pernas $(51,9 \%)$, pescoço $(36,4 \%)$, parte alta do dorso $(35,7 \%)$ e ombros (33,8\%). Analisando-se as regiões em conjunto verifica-se que $65,6 \%$ dos indivíduos referiram DORT nos membros inferiores (coxa, joelho, perna, tornozelo 
Tabela 2 - Características ocupacionais e de trabalho doméstico de técnicas e auxiliares de enfermagem. Salvador, 2008.

Table 2 - Occupational and domestic work characteristics of nursing assistants. Salvador, 2008.

\begin{tabular}{lc}
\hline Variáveis & Frequência \\
\hline Turno de trabalho n(\%) & $174(56,5)$ \\
Diurno & $78(25,3)$ \\
Noturno & $56(18,2)$ \\
Misto & \\
Hora extra n(\%) & $78(25,6)$ \\
Sim & $229(74,4)$ \\
Não & \\
Possui outro trabalho n(\%) & $203(65,9)$ \\
Não & $105(34,1)$ \\
Sim & $87(82,9)$ \\
TAE & $18(17,1)$ \\
Outros & \\
Ocupações anteriores n(\%) & $194(63,0)$ \\
Sempre atuou como TAE & $114(37,0)$ \\
Outras ocupações & $26(22,8)$ \\
$\quad$ Saúde & $31(27,2)$ \\
$\quad$ Comércio & $15(13,2)$ \\
$\quad$ Educação & $12(10,5)$ \\
$\quad$ Indústria & $30(26,3)$ \\
$\quad$ Outros & $19 \pm 10,7$ \\
Tempo total de trabalho formal+informal (anos, média $\pm d p)$ & $32 \pm 14,5$ \\
Horas de trabalho no hospital na última semana (média $\pm d p)$ & $18 \pm 15,3$ \\
Horas de trabalho doméstico na última semana (média $\pm d p)$ & \\
\hline
\end{tabular}

Tabela 3 - Distribuição dos escores para controle, demanda psicológica, suporte social e insatisfação no trabalho, por setor de trabalho. Salvador, 2008.

Table 3 -Distribution of control, psychological demand, social support and job satisfaction scores by work sector. Salvador, 2008.

\begin{tabular}{lcccc}
\hline \multirow{2}{*}{ Setores } & \multicolumn{4}{c}{ Variáveis } \\
\cline { 2 - 5 } & Controle & $\begin{array}{c}\text { Demanda } \\
\text { psicológica }\end{array}$ & $\begin{array}{c}\text { Suporte } \\
\text { Social }\end{array}$ & $\begin{array}{c}\text { Insatisfação no } \\
\text { trabalho }\end{array}$ \\
\hline UTI $(\mathrm{n}=44)$ & 64,2 & 30,4 & 22,5 & 0,17 \\
Emergência $(\mathrm{n}=68)$ & 65,8 & 32,4 & 22,3 & 0,18 \\
Centro Cirúrgico $(\mathrm{n}=35)$ & 64,2 & 34,0 & 22,1 & 0,24 \\
Enfermarias $(\mathrm{n}=57)$ & 65,7 & 32,2 & 22,9 & 0,37 \\
CME $(\mathrm{n}=20)$ & 60,3 & 30,4 & 22,8 & 0,23 \\
CTQ $(\mathrm{n}=26)$ & 64,8 & 28,6 & 24,7 & 0,09 \\
\hline
\end{tabular}

Limites Max - Mín: Controle: 96 - 24 / Demanda psicológica: 48 - 12 / Suporte social: 32 - 8 / Insatisfação no trabalho: 1 - 0 Max-Min Limits: Control: 96-24 / Psychological Demand:48 - 12/ Social Support: 32 - 8/ Job Satisfaction: 1- 0

ou pé), $57,1 \%$ referiram DORT em pescoço, ombro ou parte alta do dorso e $32,8 \%$ em extremidades superiores distais (cotovelo, antebraço, punho, mão ou dedos) (Tabela 4).
De acordo com os setores, levando-se em conta aqueles com maior número de entrevistadas, constatou-se a mais elevada prevalência dos DORT em alguma região 
Tabela 4 - Prevalência de distúrbios osteomusculares relacionados ao trabalho, segundo segmento corporal referido. Salvador, 2008.

Table 4 - Prevalence of work-related musculoskeletal disorders by body segment. Salvador, 2008.

\begin{tabular}{lcc}
\hline & \multicolumn{2}{c}{ DORT } \\
\cline { 2 - 3 } Segmento corporal referido & NIM & $\%$ \\
\hline Em alguma região (membros superiores ou inferiores ou coluna) & 257 & 83,4 \\
Pescoço, ombro ou parte alta do dorso & 176 & 57,1 \\
Extremidades superiores distais (cotovelo, antebraço, punho, mãos ou dedos) & 101 & 32,8 \\
Membros inferiores (coxa, joelho, perna, tornozelo ou pé) & 202 & 65,6 \\
Pescoço & 112 & 36,4 \\
Ombro & 104 & 33,8 \\
Cotovelo ou antebraço & 40 & 13,0 \\
Punho ou mão ou dedos & 83 & 26,9 \\
Parte alta do dorso & 110 & 35,7 \\
Lombar & 166 & 53,9 \\
Coxa ou joelho & 91 & 29,5 \\
Perna & 160 & 51,9 \\
Tornozelo ou pé & 97 & 31,5 \\
\hline
\end{tabular}

corporal nas trabalhadoras do CME (90\%), seguido pelo Centro Cirúrgico (85,7\%).

\section{Discussão}

Verificou-se uma elevada prevalência de DORT na população estudada $(83,4 \%)$. Este achado corrobora outros estudos que já demonstraram alta magnitude de DORT entre profissionais de enfermagem. Gurgueira et al. ${ }^{16}$ revelaram, em estudo com TAE que trabalhavam em unidades de internação de um hospital que atende pacientes com alto grau de dependência física, que $93 \%$ das participantes referiram algum tipo de sintoma osteomuscular nos últimos 12 meses. Smith et al. ${ }^{6}$ encontraram uma prevalência geral de DORT de $70 \%$ entre trabalhadoras de enfermagem na China, Fonseca \& Serranheira ${ }^{4}$ de $84 \%$ entre enfermeiros de Portugal, e Smith et al. ${ }^{7}$ de $85,5 \%$ entre enfermeiras no Japão.

Deve-se fazer uma ressalva quanto à comparação desses resultados, pois, apesar desses outros estudos também terem utilizado o NMQ como instrumento para coleta de dados, eles não citam a adoção dos critérios de gravidade que foram utilizados neste estudo para a definição de "caso de DORT". Neste estudo incluíram-se apenas aqueles sintomas que, além de terem duração mínima de uma semana ou frequência mínima mensal, motivassem a busca de atenção médica pelo problema ou ausência ao trabalho (oficial ou não), ou mudança de trabalho por restrição de saúde ou que tivessem um grau de severidade maior ou igual a três (em uma escala numérica de zero a cinco). A adoção desses critérios de gravidade, recomendados por Kuorinka \& Forcier $^{15}$, visou o aumento da especificidade da queixa e permite apontar com maior propriedade a grande morbidade que os DORT representam nessa população.

Comparando-se a prevalência de DORT entre os profissionais de enfermagem com os resultados de investigações realizadas com outras categorias profissionais no País, como o realizado por Brandão et al. ${ }^{17}$, que estudaram bancários em Pelotas-RS (60\%); Fernandes ${ }^{8}$, em estudo com industriários em Salvador-BA (50,1\%); e Santos Filho \& Barreto $^{18}$, com cirurgiões-dentistas em Belo Horizonte-MG (58\%), ressalvando-se as diferenças entre essas populações, como tempo de trabalho e setor (público ou privado), conclui-se que os trabalhadores de enfermagem estão entre os profissionais com maior acometimento por DORT.

Os achados deste estudo reafirmam a importância dos DORT na região lombar 
em profissionais de enfermagem. Quando foram consideradas as regiões do corpo de forma isolada verificou-se que a região lombar foi a principal acometida. O resultado deste estudo para a região lombar é comparável ao encontrado por Ando et al. ${ }^{1}(54,7 \%)$ e Smith et al. ${ }^{6}(56,7 \%)$ no Japão e China, respectivamente. Gurgueira et al. ${ }^{16}$ revelam uma prevalência um pouco mais elevada de lombalgia entre TAE em São Paulo (59\%), enquanto Fonseca \& Serranheira ${ }^{4}$ demonstram uma prevalência de $65 \%$ de DORT em região lombar em enfermeiros portugueses.

Apesar da lombar sempre ser destacada como a principal região acometida em profissionais de enfermagem, verifica-se que quando foram consideradas na análise as regiões do pescoço, ombro e parte alta do dorso conjuntamente, como já realizado em outros trabalhos ${ }^{5,8}$, os sintomas nessa região foram mais prevalentes $(57,1 \%$, quando comparados a 53,9\% na região lombar). Essa análise conjunta de pescoço, ombro e parte alta do dorso pode ser adotada, pois se considera que estes segmentos atuam como uma unidade funcional. É difícil identificar especificamente os fatores de exposição e sintomas para essas áreas separadamente ${ }^{19}$.

Surpreende a magnitude de DORT relatada em membros inferiores. Verifica-se que a principal região que contribui para este resultado são as pernas, que, isoladamente, correspondem a 51,9\%. Gurgueira et al. ${ }^{16}$ encontraram a prevalência de DORT em joelhos apenas menor que a prevalência de DORT na região lombar e ombros em trabalhadoras de enfermagem. Fernandes ${ }^{8}$ aponta uma prevalência de $21,3 \%$ de DORT em região lombar e $18,2 \%$ em pernas, tornozelo ou pé em trabalhadores industriais.

Sheikhzadeh et al. ${ }^{20}$ revelam dados importantes em relação à DORT em membros inferiores em enfermeiras e técnicas de enfermagem de um centro cirúrgico. Nesse estudo, conduzido nos Estados Unidos, a maior prevalência de DORT encontrada nos sete dias anteriores à entrevista foi na região de tornozelo/pé (59\%). Queixas de dor em tornozelo/pé foram a segunda causa de absenteísmo ao trabalho (24\%), apenas atrás da região lombar (31\%) e seguida por dor em joelhos (21\%). Segundo os autores, ficar em pé por longas horas (mais de 10 horas em alguns procedimentos cirúrgicos) é um contribuinte importante para dor em lombar e tornozelo/pé.

Apesar de poucos estudos abordarem DORT em membros inferiores, quando estes são considerados nas análises, têm mostrado prevalências bastante significativas que não têm sido discutidas. Os achados deste estudo reforçam a necessidade de investigações que se dediquem ao estudo de DORT em membros inferiores.

Em relação ao auto-relato de exposição às demandas físicas no trabalho, constatou-se o resultado mais expressivo para a realização de movimentos repetitivos com as mãos. Este achado para as TAE é idêntico ao verificado para trabalhadoras da indústria de plástico, que utilizou o mesmo instrumento para a coleta de dados ${ }^{8}$.

O levantamento de cargas referido pelas TAE, também bastante frequente, em boa parte representa as atividades ligadas à transferência de pacientes. No entanto, também se observam outras atividades não ligadas diretamente aos pacientes, que também exigem levantamento de carga. No CME, por exemplo, onde não há contato direto com pacientes, foi relatada alta frequência de manuseio de cargas, a qual está relacionada com manuseio e transporte de materiais e equipamentos.

Os resultados obtidos mostram que as TAE mantêm o tronco inclinado para frente em boa parte da sua jornada de trabalho, o que pode ser explicado pelo fato de que os cuidados são prestados a pacientes que geralmente estão acamados. Isto é confirmado pelo achado de que a elevação dos braços acima da altura dos ombros só ocorre ocasionalmente.

Neste estudo, em relação à demanda psicossocial de acordo com os setores de trabalho, o maior controle foi encontrado na emergência, seguido das enfermarias. Quanto à demanda psicológica, esses dois setores também apresentaram escores importantes, ficando apenas atrás do centro 
cirúrgico. Foi encontrada uma diferença estatisticamente significante ( $p$ valor $<0,05$ ) entre os escores para a demanda psicológica entre os profissionais das enfermarias e UTI, com maior escore para a enfermaria. Apesar de as TAE da UTI lidarem com pacientes com quadros mais graves, esses estão monitorizados e a unidade dispõe de uma equipe multiprofissional 24h. Já nas enfermarias, não há aparelhos para o monitoramento dos pacientes, o que faz com que as TAE tenham de estar mais atentas aos sinais e sintomas dos pacientes para que possa ser verificada alguma alteração. Além disso, as enfermarias não contam com médicos em tempo integral. Caso haja intercorrência com algum paciente, são os profissionais do setor que têm de prestar os primeiros socorros até a chegada dos demais membros da equipe multiprofissional. Isso se agrava no período noturno, no qual os únicos profissionais no setor são os de enfermagem.

Guedes et al..$^{22}$, ao fazerem a análise ergonômica de uma das principais atividades dos auxiliares de enfermagem, a medicação, mostram a alta demanda psicológica envolvida nessa atividade, que implica grande responsabilidade, uma vez que um erro pode trazer consequências graves para o paciente, exigência de conhecimentos específicos e, muitas vezes, pressão temporal devido ao atraso na disponibilização da medicação. Esses autores também mostram um grau de controle desses profissionais em relação a essa atividade, quando revelam as suas iniciativas para a adoção de estratégias para garantir a qualidade do tratamento $\mathrm{e}$ minimizar o sofrimento do paciente.

O centro cirúrgico, apesar do maior escore para a demanda psicológica, apresentou o escore mais baixo em relação ao suporte social. Estes achados podem justificar por que o centro cirúrgico ficou apenas atrás das enfermarias no quesito insatisfação no trabalho.

O principal setor acometido por DORT neste estudo foi o CME. Apesar de não lidarem diretamente com pacientes, as TAE desse setor realizam tarefas repetitivas e com manuseio de carga, e possuem baixo controle sobre o seu trabalho. Castro ${ }^{23}$ ressalta a problemática relacionada às questões ergonômicas em CME, colocando que, em geral, esses setores são instalados em áreas pequenas, não adaptadas aos trabalhadores, cujos móveis e máquinas também não seguem os princípios da ergonomia. Desta forma, conclui que é comum encontrar trabalhadores de CME com DORT, principalmente lombalgias, sendo estas as mais encontradas em publicações sobre saúde do trabalhador dessa área ${ }^{23}$.

Este estudo, assim como outros, cuja população alvo são profissionais de enfermagem $^{6,7,16}$, trabalhou apenas com a população feminina. Esta opção metodológica decorre do pequeno número de homens inseridos na enfermagem. Ao estudar trabalhadoras, é importante considerar as atividades que estas desempenham em casa. A divisão sexual do trabalho doméstico e do cuidado aos filhos, apesar de todas as mudanças sociais, continua exigindo maior participação das mulheres. Neste estudo, as mulheres dedicavam, em média, 18 horas semanais ao trabalho doméstico.

Aquino $^{21}$, em estudo realizado com profissionais de enfermagem, evidencia que as mulheres, em sua maioria, possuíam duplo emprego e, mesmo assim, não estavam liberadas do trabalho doméstico. O tempo despendido com as atividades domésticas soma-se ao da jornada de trabalho assalariado, invadindo o tempo livre para o descanso e o lazer.

\section{Considerações finais}

A magnitude de DORT, encontrada entre as profissionais estudadas, bem como a descrição das demandas físicas e psicossociais às quais as TAE estão expostas, sinalizam para a necessidade de atenção a essa categoria profissional e às suas condições de trabalho. Tendo em vista o potencial evolutivo dos quadros do DORT e a possibilidade de surgimento de novos casos, ressalta-se a necessidade da aplicação do conhecimento já produzido para a melhoria das condições de trabalho dessas profissionais. 


\section{Referências}

1. Ando S, Ono Y, Shimaoka M, Hiruta S, Hattori Y, Hori et al. Associations of self estimated workloads with musculoskeletal symptoms among hospital nurses. Occup Environ Med 2000; 57: 211-16.

2. Brasil. Ministério da Saúde. Lesões por esforços repetitivos (Ler) e Distúrbios osteomusculares relacionados ao trabalho (DORT). Brasília: Ministério da Saúde; 2001.

3. Smith DR, Leggat PA. Musculoskeletal disorders in nursing. Aust Nurs J 2003; 11: 1-4.

4. Fonseca R, Serranheira F. Sintomatologia musculoesquelética autorreferida por enfermeiros em meio hospitalar. Rev Port Saude Publica 2006; 6: 37-44.

5. Smedley J, Inskip H, Trevelyan F, Buckle P, Cooper C, Coggon D. Risk factors for incident neck and shoulder pain in hospital Nurses. Occup Environ Med 2003; 60: 864-9.

6. Smith DR, Wei N, Zhao L, Wang RS. Musculoskeletal complaints and psychosocial risk factors among Chinese hospital nurses. Occup Med 2004; 54: 579-82.

7. Smith DR, Mihashi M, Adachi Y, Koga H, Ishitake T. A detailed analysis of musculoskeletal disorder risk factors among Japanese nurses. J Safety Res 2006; 37: 195-200.

8. Fernandes RCP. Distúrbios musculoesqueléticos e trabalho industrial [tese de doutorado]. Salvador: Instituto de Saúde Coletiva da Universidade Federal da Bahia; 2004.

9. Araújo T. "Job Content Questionnaire" - JCQ (short version - recommended format: 49 questions), elaborado por Robert Karasek: tradução e adaptação (mimeo); 2000.

10. Alves MGM, Chor D, Faerstein E, Lopes CS, Werneck GL. Versão resumida da "Job Stress Scale": adaptação para o português. Rev Saúde Pública 2004; 38:164-71.

11. Araújo TM, Karasek R. Job Content Questionnaire: formal and informal job in a Brazilian study. Int $J$ Behavioral Medicine 2008; 11 S: 167-8.

12. Karasek R, Brisson C, Kawakami N, Bongers P, Houtman I, Amick B. The Job Content Questionnaire (JCQ): An Instrument for Internationally comparative assessments of psychosocial job characteristics. J Occup Health Psychol 1998; 3(4): 322-55.

13. Pinheiro FA, Tróccoli BT, Carvalho CV. Validação do Questionário Nórdico de Sintomas Osteomusculares como medida de morbidade. Rev Saúde Públ 2002; 36(3): 307-12.
14. Barros ENC, Alexandre NMC. Cross-cultural adaptation of the Nordic muloskeletal questionnaire. International Nursing Review 2003; 50(2): 101-8.

15. Kuorinka I, Forcier L. (scientific editors). Work related musculoskeletal disorders (WMSDs): a reference book for prevention. London: Taylor \& Francis; 1995.

16. Gurgueira GP, Alexandre NMC, Corrêa HR. Prevalência de sintomas musculoesqueléticos em trabalhadoras de enfermagem. Rev Latino-am Enfermagem 2003; 11(5): 608-13.

17. Brandão AG, Horta BL, Tomasi E. Sintomas de Distúrbios osteomusculares em bancários de Pelotas e região: prevalência e fatores associados. Rev Bras Epidemiol 2005; 8(3): 295-305.

18. Santos Filho SB, Barreto SM. Atividade ocupacional e prevalência de dor osteomuscular em cirurgiõesdentistas de Belo Horizonte, Minas Gerais, Brasil: contribuição ao debate sobre os distúrbios osteomusculares relacionados ao trabalho. Cad Saúde Pública 2001; 17(1): 181-93.

19. National Research Council \& Institute of Medicine [NRC \& IM]. Musculoskeletal disorders and the workplace: low back and upper extremities. Panel on musculoskeletal disorders and the workplace. Commission on behavioral and social sciences and education. Washington, DC: National Academy Press; 2001.

20. Sheikhzadeh A, Gore C, Zuckerman JD, Nordin M. Perioperating nurses and technicians' perceptions of ergonomic risk factors in the surgical environment. Appl Ergon 2008 [in press].

21. Aquino EML. Gênero, trabalho e hipertensão arterial: um estudo de trabalhadoras de enfermagem em Salvador, Bahia [tese de doutorado]. Salvador: Instituto de Saúde Coletiva da Universidade Federal da Bahia; 1996.

22. Guedes RMA, Lima FPA, Assunção AA. O programa de qualidade no setor hospitalar e as atividades reais da enfermagem: o caso da medicação. Ciênc Saúde Colet 2005; 10(4): 1063-704.

23. Castro MES. Condições de trabalho e fatores de risco à saúde dos trabalhadores do centro de material esterilizado do Hospital de clínicas da UFPR [dissertação de mestrado]. Florianópolis: Programa de PósGraduação em Engenharia da Produção/UFSC; 2002.

Recebido em: 12/09/2009 Versão final apresentada em: 09/08/2011 Aprovado em: 03/09/2011 